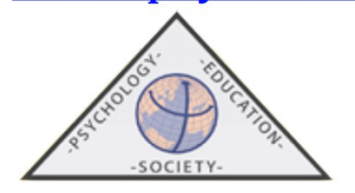

\title{
Salud mental y consumo de drogas en prisiones españolas. Una perspectiva socioeducativa y de género
}

\author{
Diego GALÁN CASADO ${ }^{1}$, Eva Ma RAMOS-ÁBALOS ${ }^{2}$, Ángel TURBI-PINAZO

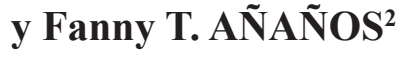 \\ ${ }^{1}$ Universidad Camilo José Cela \\ ${ }^{2}$ Universidad de Granada \\ ${ }^{3}$ Universidad Católica de Valencia
}

(Recibido el 10 de Mayo de 2020, Aceptado el 29 de Julio de 2020)

\begin{abstract}
RESUMEN: El trabajo tiene como objeto analizar la relación existente entre las distintas situaciones y autopercepciones asociadas a los problemas de salud mental con el consumo de drogas, en una investigación cualitativa y cuantitativa, teniendo como muestra nacional encuestada de 310 (30,1\%) mujeres reclusas en semilibertad. Los análisis fueron estadísticos descriptivos, contrastes de independencia, test de Fisher y odds-ratio. Los resultados indican que el 73,2\% ha sentido/padecido algún problema de salud mental, siendo pocas las diagnosticadas y recibieron tratamientos fundamentalmente farmacológicos; asimismo el 51,6\% ha consumido drogas. La participación en programas específicos es muy baja. Todas estas situaciones instan a la mejora de la intervención penitenciaria, la necesidad de implementar y fomentar la acción socioeducativa, mediante programas integrales que favorezcan la mayor participación, con contenidos coherentes a la realidad y desde el enfoque de género, los cuales incidirán en la reinserción social.
\end{abstract}

Palabras clave: drogas, salud mental, prisión, genero, reinserción social, Intervención socioeducativa.

\section{Mental Health and Drug Use in Spanish Prisons. A Socio-Educational and Gender Perspective}

ABSTRACT: The aim of the work is to analyse the relationship between different situations and selfperceptions associated with mental health problems and drug use, in a qualitative and quantitative research, having as a national sample surveyed 310 (30.1\%) women prisoners in semi-freedom. The analyses were: descriptive statistics, independence contrasts, Fisher test and odds ratio. The results indicate that $73.2 \%$ have felt/suffered some mental health problem, with few being diagnosed and receiving mainly pharmacological treatment; likewise $51.6 \%$ have consumed drugs. Participation in specific programmes is very low. All these situations call for improved prison intervention and the need to implement and promote socio-educational action through comprehensive programmes that encourage greater participation, with content that is consistent with reality and from a gender perspective, which will have an impact on social reintegration.

Keywords: drugs, mental health, prison, gender, social reintegration, socio-educational intervention. 
Correspondencia: Fanny T. Añaños. Campus Universitario de Cartuja, 18071 Granada. E-mail: fanntab@ugr.es

\section{Introducción}

La pérdida de libertad es un proceso complejo y muy difícil para la persona, donde se dan múltiples cambios, entre otros, en la vida personal, familiar, social, además de la adecuación a la vida en prisión. Todo ello puede generar diversos problemas y dificultades, especialmente en el afrontamiento individual y emocional de esta situación, por lo que el objetivo de la investigación pretende analizar la relación existente entre las distintas situaciones y autopercepciones asociadas a la problemática de salud mental, la relación con las sustancias $\mathrm{y}$, la asociación de ambas, en el que la perspectiva socioeducativa esté presente.

En primer lugar, a pesar de que no existe una definición generalizada sobre el Trastorno Mental Grave -en adelante TMG- (Boysen, 2007), la OMS (2020) refiere que se caracteriza por una combinación de alteraciones del pensamiento, la percepción, las emociones, la conducta y las relaciones con los demás. Sin embargo, es necesario distinguir la diferencia entre síntoma o diagnóstico, aunque parecen ir de la mano. Así, los síntomas son manifestaciones patológicas subjetivas, es decir, aquellas que un especialista no puede percibir (cansancio, nauseas...), siendo el signo la manifestación objetiva, evidente y tangible derivado de un examen físico o mental de la persona (Ezama, Alonso y Fontanil, 2010; Gil Extremera, 2019). En cambio, el diagnóstico viene a ser la asignación o identificación de esas manifestaciones concretas (Coronado, 2016), donde un profesional de la salud identifica una determinada enfermedad, trastorno o síndrome. El diagnóstico en este ámbito resulta complicado debido a la falta de límites claros entre las diversas patologías (Vega-Dienstmaier, 2017), al que hay que sumar la sintomatología percibida.

Centrándonos en el medio penitenciario, podemos resaltar que, según Turbi y LLopis, (2017), los trastornos del estado de ánimo (depresión y ansiedad), junto a los cognoscitivos (problemas de memoria, concentración, etc.) son los más frecuentes en mujeres, así como los de tipo paranoide (Herrera, 2000), en el que los/as internos/as se sienten constantemente perseguidos o amenazados. Asimismo, el consumo de psicofármacos en prisión, llega incluso a triplicarse si lo comparamos con la población exterior (Espinosa y Laliga, 2005), donde, además, la atención a la salud mental suele realizarse en los módulos de enfermería, por personal sanitario no especializado y, en el caso de existir, en el módulo terapéutico de la prisión. A todo se añade que son limitados los casos de cumplimiento de condena en centros especializados como centros penitenciarios psiquiátricos (existen 2 en toda España) o algunas unidades terapéuticas en instituciones hospitalarias.

La intervención socioeducativa y/o psicoeducativa dentro de prisión se da mediante el programa PAIEM (Programa de Atención Integral a Internos con Enfermedad Mental). Dicho programa, se estructura en torno a tres ejes: detección del caso y atención clínica, programa de rehabilitación individualizado e incorporación social (Secretaría General de Instituciones Penitenciarias-en adelante SGIP-, 2020).

En segundo lugar, sobre la adicción a las drogas, conocida también como «trastorno por consumo de sustancias»-TUS- (American Psychiatric Association, 2014), se considera una en- 
fermedad que, entre otros, afecta al funcionamiento fisiológico, sistema nervioso central, comportamiento, y produce, incapacidad de controlar el consumo de medicamentos o drogas. En ella es importante diferenciar subgrupos, de tal modo que se definen perfiles o rasgos, dadas las características concretas en cada una, así Añaños (2017) y Añaños, Fernández y LLopis, (2013); distingue cuatro subgrupos: Adictas Activas (AA), quienes presentan consumo actual; Adictas en Programas de Metadona (PMM; Ex Adictas (EX), abstinentes al menos 6 meses previos a la investigación y; No adictas (NA), quienes no presentan consumos problemáticos de alcohol y drogas.

Ruiz Naredo y Santibañez Gruber (2014) indican que son consumidores aproximadamente la tercera parte de los hombres adultos en prisiones de la Unión Europea y, en el caso de España la cifra es del 50\%. En un estudio nacional español, en 42 prisiones, se llega a identificar al 60,6\% de mujeres consumidoras, distribuidas en los distintos grupos de adicción -AA, PMM y EX-,en el momento de ingresar a prisión (Añaños, 2017). Datos que concuerdan con la encuesta sobre consumo de drogas en prisión -ESDIP-, que refiere que los consumos de todas las drogas ilegales son claramente más elevados en la población penitenciaria (estando en libertad) que, en la población general, siendo las más consumidas: alcohol (65,9\%), cannabis $(37,8 \%)$ y cocaína en polvo $(24,8 \%)$ y en base $(16,2 \%)$ (Ministerio de Sanidad, Servicios Sociales e Igualdad, 2016).

En tercer lugar, y una vez abordados las dos cuestiones de manera independiente, se centra el análisis en la asociación de ambas, que se denomina "patología dual", siendo ésta una realidad en la que concurren uno o varios trastornos psiquiátricos y, a la vez, una patología adictiva (Casas y Guardia, 2002). Así encontramos disparidad en los datos hallados: Villagrá et al (2011), en un estudio de casos con 59 mujeres encuentra a 64,4\% consumidoras, de las cuales el 47,4\% presentaba patología dual; otros trabajos que no distinguen sexo de la muestra indican, un 29\% (Butler et al., 2011) y un 26,3\% (Lukasiewicz et al., 2009). Finalmente, Baillargreon et al. (2010), expresan que la mayor prevalencia de patología dual en prisión se da en mujeres.

\section{Método}

Los datos que a continuación se analizan corresponden a los resultados del Proyecto de Investigación $\mathrm{I}+\mathrm{D}+\mathrm{i}-R E I N A C$ - "Procesos de reinserción y acompañamiento a mujeres en semilibertad" (Ref. EDU2016-79322-R), avalada por la SGIP y la Consejería de Justicia de la Generalitat de Catalunya, cuyos Comités Éticos han aprobado la investigación.

La población está compuesta por mujeres en cumplimento de condena en medio abierto español (3er grado y 2do grado especial), en una muestra válida de 310 (30,1\% de la población femenina), en 31 entidades penitenciarias de 13 Comunidades Autónoma. El muestreo fue bietápico, por representación territorial y ratio de mujeres y posteriormente al azar entre aquellas que querían participar voluntariamente.

El instrumento empleado fue un cuestionario mixto, diseñado Ad hoc. El cuestionario en su totalidad tuvo 115 preguntas abiertas y cerradas, de las que se seleccionaron las variables asociadas al objeto del presente trabajo. Se aplicó tras firmar el consentimiento informado y, en función de las competencias lectoras o conocimiento del idioma se realizó en pequeños grupos o individualmente. 
En el análisis de la información cualitativa se empleó el método de análisis de contenido, lo que posteriormente permitió cuantificar la dimensión de las autopercepciones y realizar los otros tipos de análisis. Por otro, los métodos de análisis cuantitativos han combinado frecuencias, contingencias, contrastes de independencia: test chi-cuadrado con corrección por continuidad y test exacto de Fisher en el caso de que no haya sido válido el test chi-cuadrado. En las relaciones significativas, se ha calculado la razón de ventajas (odds-ratio) como medida de asociación.

\section{Resultados}

Los resultados obtenidos, han sido estructurados en tres epígrafes concretos (salud mental, relación con las sustancias y el consumo de drogas y la salud mental) con el propósito de organizar mejor la información.

\section{La salud mental}

Tabla 1. Situación de salud mental

\begin{tabular}{lcccccc}
\hline & \multicolumn{2}{c}{ Sintomatología percibida } & \multicolumn{2}{c}{ Recibe tratamiento } & \multicolumn{2}{c}{ Diagnóstico salud mental } \\
& $\mathbf{N}$ & $\mathbf{\%}$ & $\mathbf{N}$ & $\mathbf{\%}$ & $\mathbf{N}$ & $\mathbf{\%}$ \\
\hline No & 70 & $22,6 \%$ & 146 & $64.3 \%$ & 43 & $53.1 \%$ \\
$\mathrm{Si}$ & 227 & $73,2 \%$ & 81 & $35.7 \%$ & 37 & $45.7 \%$ \\
En blanco & 13 & $4,2 \%$ & - & - & 1 & $1.2 \%$ \\
Totales & 310 & $100 \%$ & 227 & 100 & 81 & 100 \\
\hline
\end{tabular}

Fuente: Elaboración propia a partir del proyecto REINAC Ref. EDU2016-79322-R.

Se inicia el análisis situando que casi tres cuartas partes del total de mujeres estudiadas $(73,2 \%, N=310)$ ha sentido o percibido algún problema de salud mental -en adelante sintomatología percibida-, y de éstas $37(16,3 \%, \mathrm{~N}=227)$, que representa $11,9 \%$ de la muestra total, tiene algún diagnóstico de salud mental. Sin embargo, la mayor parte de las que han tenido el problema no han recibido ningún tratamiento $(64,3 \%, \mathrm{~N}=227)$.

En el grupo de mujeres con sintomatología autopercibida y que tienen algún tratamiento $(35,7 \%)$, algo más de la mitad $(53,1 \%)$ no tienen diagnóstico de salud mental frente al $45,7 \%$ que tiene un diagnóstico. El tratamiento más frecuente recibido es el farmacológico $(34,4 \%)$ y el psicológico (12,3\%). 
Tabla 2. Sintomatología de salud mental autopercibida

\begin{tabular}{|c|c|c|c|c|}
\hline \multirow{2}{*}{ Situaciones autopercibidas } & \multicolumn{2}{|c|}{ SI } & \multicolumn{2}{|c|}{ NO } \\
\hline & $\mathbf{N}$ & $\%$ & $\mathbf{N}$ & $\%$ \\
\hline Me encuentro deprimida, triste y vacía sin causa o motivo aparente o fuerte & 139 & $44,8 \%$ & 157 & $50,6 \%$ \\
\hline $\begin{array}{l}\text { Suelo estar nerviosa, ansiosa, intranquila, tensa, etc. con miedo o temor } \\
\text { continuo }\end{array}$ & 133 & $42,9 \%$ & 161 & $51,9 \%$ \\
\hline Me olvido con facilidad las cosas o me cuesta concentrarme & 131 & $42,3 \%$ & 162 & $52,3 \%$ \\
\hline Escucho voces en mi cabeza o he oído voces sin que haya nadie alrededor & 31 & $10,0 \%$ & 263 & $84,8 \%$ \\
\hline Siento que me espían o persiguen & 39 & $12,6 \%$ & 253 & $81,6 \%$ \\
\hline $\begin{array}{l}\text { Cuando me enfado o no consigo lo que quiero, me salen insultos, rompo } \\
\text { cosas, amenazo o agredo a alguien. }\end{array}$ & 26 & $8,4 \%$ & 267 & $86,1 \%$ \\
\hline 1 En alguna ocasión me han diagnosticado la personalidad: Antisocial & 10 & $3,2 \%$ & 277 & $89,4 \%$ \\
\hline 7.2 En alguna ocasión me han diagnosticado la personalidad: Límite & 4 & $1,3 \%$ & 282 & $91,0 \%$ \\
\hline 7.3 En alguna ocasión me han diagnosticado la personalidad: Histriónico & 2 & $0,6 \%$ & 284 & $91,6 \%$ \\
\hline $\begin{array}{l}\text { He perdido peso mediante dietas exageradas, ayunando/haciendo ejercicios } \\
\text { duros }\end{array}$ & 50 & $16,1 \%$ & 241 & $77,7 \%$ \\
\hline Hay periodos que tras meterme atracones de comida me siendo mal y vomito & 27 & $8,7 \%$ & 266 & $85,8 \%$ \\
\hline $\begin{array}{l}\text { 10. Alguna vez he realizado alguna cosa para morirme y terminar mi } \\
\text { sufrimiento }\end{array}$ & 41 & $13,2 \%$ & 250 & $80,6 \%$ \\
\hline 11. Alguna vez me he golpeado o lesionado para hacerme daño a mí misma & 34 & $11,0 \%$ & 258 & $83,2 \%$ \\
\hline 12. Otra sensación o situación & 7 & $2,3 \%$ & 0 & $50,6 \%$ \\
\hline Totales & 310 & 100 & 310 & 100 \\
\hline
\end{tabular}

Fuente: Elaboración propia a partir del proyecto REINAC Ref. EDU2016-79322-R.

La Tabla N 2 analiza las respuestas de forma independiente. La sintomatología más frecuente está relacionada con estados depresivos (44,8\%), seguida de ansiedad (42,9\%), falta de concentración y olvidos $(42,3 \%)$. También, se observan cuadros relacionados a trastornos de la alimentación: anorexia $(16,1 \%)$ y bulimia $(8,7 \%)$, así como situaciones relacionadas con intentos de suicidio $(13,2 \%)$ y procesos autolíticos $(11 \%)$. Igualmente es llamativa las situaciones asociadas a la esquizofrenia- obsesión/compulsión $(12,6 \%)$ y, alucinaciones auditivas y persecutorias (10\%). Finalmente, los datos más bajos, responden a mujeres con algún diagnóstico propio de trastornos de la conducta (histriónico, antisocial y límite).

\section{Relación con las sustancias}

Para la definición de los subgrupos de consumo de sustancias y la adicción, se siguió los criterios planteados por Añaños (2017) y Añaños, Fernández y Llopis (2013), en los que excluyen las siguientes sustancias: tabaco, alcohol de uso y tranquilizantes recetados. Así, algo más de la mitad de las mujeres estudiadas $(\mathrm{N}=160 ; 51,6 \%)$ ha consumido alguna sustancia (en adelante "consumidoras"). Sin embargo, sólo 72 (45\%) de éstas reconocen/expresan que han tenido o tienen 
una adicción, representando el 23,2\% en la muestra total. Por otro lado, las adictas en un 59,7\% han recibido un tratamiento para dejar/controlar el consumo de drogas, realizándolo en prisión $(25,6 \%)$ como actualmente $(11,6 \%)$,siendo más frecuente el tratamiento en Programas de Mantenimiento de Metadona-PMM- (14\%), seguido de prevención de recaídas (9,3\%), Comunidad Terapéutica (7\%), desintoxicación (4,7\%) y ambulatorio (2,3\%).

\section{El consumo de drogas y la salud mental}

Tabla 3. Relación entre la sintomatología autopercibida con el consumo de drogas y la adicción

\begin{tabular}{|c|c|c|c|c|c|}
\hline \multirow{2}{*}{ No } & & & \multicolumn{2}{|c|}{ Consumo sustancias } & \multirow{2}{*}{ Total } \\
\hline & & & Sí & & \\
\hline \multirow{6}{*}{$\begin{array}{l}\text { Ahora, Sintomatología } \\
\text { autopercibida }\end{array}$} & \multirow[t]{2}{*}{ No } & Recuento & 39 & 31 & 70 \\
\hline & & Consumo sustancias & $27,7 \%$ & $19,9 \%$ & $23,6 \%$ \\
\hline & \multirow[t]{2}{*}{ Sí } & Recuento & 102 & 125 & 227 \\
\hline & & Consumo sustancias & $72,3 \%$ & $80,1 \%$ & $76,4 \%$ \\
\hline & \multirow[t]{2}{*}{ Total } & & 141 & 56 & 297 \\
\hline & & Consumo sustancias & $100,0 \%$ & $100,0 \%$ & $100,0 \%$ \\
\hline \multirow{2}{*}{ No } & & & \multicolumn{2}{|c|}{ Adicción } & \multirow{2}{*}{ Total } \\
\hline & & & Sí & & \\
\hline \multirow{6}{*}{$\begin{array}{l}\text { Ahora, Sintomatología } \\
\text { autopercibida }\end{array}$} & \multirow[t]{2}{*}{ No } & \multirow{2}{*}{$\begin{array}{l}\text { Recuento } \\
\text { Adicción }\end{array}$} & 19 & 12 & 31 \\
\hline & & & $22,4 \%$ & $16,9 \%$ & $19,9 \%$ \\
\hline & \multirow[t]{2}{*}{ Sí } & \multirow{2}{*}{$\begin{array}{l}\text { Recuento } \\
\text { Adicción }\end{array}$} & 66 & 59 & 125 \\
\hline & & & $77,6 \%$ & $83,1 \%$ & $80,1 \%$ \\
\hline & \multirow[t]{2}{*}{ Total } & \multirow{2}{*}{ Adicción } & 85 & 71 & 156 \\
\hline & & & $100,0 \%$ & $100,0 \%$ & $100,0 \%$ \\
\hline
\end{tabular}

Fuente: Elaboración propia a partir del proyecto REINAC Ref. EDU2016-79322-R.

En la muestra total $(\mathrm{N}=310)$, encontramos un 40,32\% $(\mathrm{N}=125)$ de mujeres consumidoras de drogas y que, a la vez, tienen alguna sintomatología de salud mental y, un 19,03\% (N=59) de mujeres adictas con sintomatología.

Concretamente, en el grupo consumidoras, el $80,1 \%$ indica que ha sentido o padecido algún problema de salud mental, un 7,8\% (80,1\%-72,3\%) más que en el grupo de no consumidoras. En el caso de las adictas, el 83,1\% indica que ha tenido algún problema de salud mental, un 5,5\% $(83,1 \%-77,6 \%)$ más que en mujeres no adictas. 
Tabla 4. Relación entre el diagnóstico de salud mental con el consumo de drogas y la adicción

\begin{tabular}{|c|c|c|c|c|c|}
\hline $\begin{array}{l}\text { No } \\
\text { Sí }\end{array}$ & & & \multicolumn{2}{|c|}{$\begin{array}{l}\text { Consumo } \\
\text { sustancias }\end{array}$} & Total \\
\hline \multirow{5}{*}{ Diagnóstico de salud mental } & \multirow{2}{*}{ No } & \multirow[t]{2}{*}{ Recuento } & 16 & 32 & 48 \\
\hline & & & $57,1 \%$ & $56,1 \%$ & $56,5 \%$ \\
\hline & \multirow{2}{*}{ Sí } & \multirow[t]{4}{*}{ Recuento } & 12 & 25 & 37 \\
\hline & & & $42,9 \%$ & $43,9 \%$ & $43,5 \%$ \\
\hline & \multirow{2}{*}{$\begin{array}{l}\text { Recuento } \\
\text { Consumo sustancias } \\
100,0 \%\end{array}$} & & 28 & 57 & 85 \\
\hline Total & & & $100,0 \%$ & $100,0 \%$ & \\
\hline \multicolumn{2}{|l|}{$\begin{array}{l}\text { No } \\
\text { Sí }\end{array}$} & & \multicolumn{2}{|c|}{ Adicción } & Total \\
\hline \multirow{5}{*}{ Diagnóstico de salud mental } & \multirow{2}{*}{ No } & Recuento & 13 & 19 & 32 \\
\hline & & & $54,2 \%$ & $57,6 \%$ & $56,1 \%$ \\
\hline & \multirow{2}{*}{ Sí } & Recuento & 11 & 14 & 25 \\
\hline & & & $45,8 \%$ & $42,4 \%$ & $43,9 \%$ \\
\hline & \multirow{2}{*}{\multicolumn{2}{|c|}{$\begin{array}{l}\text { Recuento } \\
\text { \%Adicción } \\
100,0 \%\end{array}$}} & 24 & 33 & 57 \\
\hline Total & & & $100,0 \%$ & $100,0 \%$ & \\
\hline
\end{tabular}

Fuente: Elaboración propia a partir del proyecto REINAC Ref. EDU2016-79322-R.

En las adictas $(\mathrm{N}=72)$, sólo $14(19,4 \%)$ tienen un diagnóstico de salud mental, además de la adicción, considerándose éstas las que presentan una patología dual, un 3,4\% (42,4\%-45,8\%) menos que en el grupo de no adictas (NA). En relación a la muestra total $(\mathrm{N}=310)$ representa un escaso 4,5\%. Además, hay 6,13\% (19, N=310) de adictas no diagnosticadas.

En cuanto a la participación en programas de salud mental, sólo $8(3,5 \%, \mathrm{~N}=227)$ de las que tenía alguna sintomatología lo han realizado en prisión, constituyendo un escaso 2,6\% en la muestra total. De las 8, 4 son adictas, 2 son consumidoras y 2 no consumidoras, de éstas sólo 2 tienen diagnóstico de salud mental.

Tabla 5. Variables relacionadas significativamente

\begin{tabular}{llcc}
\hline Variable 1 & Variable 2 & p-value & Odds-ratio \\
\hline $\begin{array}{l}\text { Ahora, Sintomatología } \\
\text { de salud mental }\end{array}$ & $\begin{array}{l}\text { ¿Recibes tratamiento por alguna situación que has puesto } \\
\text { en la tabla? }\end{array}$ & $<0,0001$ & 9.154 \\
& $\begin{array}{l}\text { ¿Has consumido alguna vez o consumes tranquilizantes } \\
\text { recetados para dormir, relajarme, nervios, etc.? }\end{array}$ & $<0,0001$ & 4,875 \\
Consumo de sustancias & $\begin{array}{l}\text { ¿Has consumido alguna vez o consumes tranquilizantes } \\
\text { no recetados para dormir, relajarme, nervios, etc.? }\end{array}$ & 0,001 & 26.816 \\
\hline
\end{tabular}

Fuente: Elaboración propia a partir del proyecto REINAC Ref. EDU2016-79322-R.

A partir de las variables que han resultado significativas desglosamos las siguientes tablas descriptivas, haciendo, a la vez, el análisis de la significación (Tabla No5). 
Tabla6.Relación en tratamiento recibido y sintomatología de salud mental

\begin{tabular}{|c|c|c|c|c|c|}
\hline \multirow[t]{2}{*}{$\begin{array}{l}\text { No } \\
\text { Sí }\end{array}$} & & \multicolumn{2}{|c|}{ Ahora. Sintomatología } & \multirow{2}{*}{$\begin{array}{c}\text { Total } \\
212\end{array}$} \\
\hline & \multirow{2}{*}{ No } & Recuento & 66 & 146 & \\
\hline \multirow{4}{*}{$\begin{array}{l}\text { Tratamiento por } \\
\text { sintomatología }\end{array}$} & & $\%$ dentro de Ahora. Sintomatología & $94,3 \%$ & $64,3 \%$ & $71,4 \%$ \\
\hline & \multirow{2}{*}{ Sí } & Recuento & 4 & 81 & 85 \\
\hline & & $\%$ dentro de Ahora. Sintomatología & $5,7 \%$ & $35,7 \%$ & $28,6 \%$ \\
\hline & \multirow{2}{*}{\multicolumn{2}{|c|}{$\begin{array}{l}\text { Recuento } \\
\text { Ahora. Sintomatología } \\
100,0 \%\end{array}$}} & 70 & 227 & 297 \\
\hline Total & & & $100,0 \%$ & $100,0 \%$ & \\
\hline
\end{tabular}

Fuente: Elaboración propia a partir del proyecto REINAC Ref. EDU2016-79322-R.

La Tabla N6 muestra los porcentajes sobre el total de la columna (70 No y 227 Sí). El contraste chi-cuadrado sobre la independencia de las variables es significativo $(<0,0001)$, verificándose las hipótesis para aplicarlo (Tabla $\mathrm{N}^{\circ} 5$ ), de modo que existe una asociación positiva significativa entre recibir tratamiento y haber presentado sintomatología de salud mental. Se obtiene un cociente de ventajas de $\theta=9.154$, que indica que la probabilidad de recibir tratamiento es algo más de 9 veces superior en las mujeres con sintomatología que en las mujeres que no tienen esta situación.

Considerando un total de 297 mujeres con respuestas válidas, un 76,4\% (227 mujeres) presenta sintomatología de salud mental, de la cuales, un 64,3\% (146) no recibe ningún tratamiento frente al 35,7\% (81) que si lo recibe. Además, un 23,6\% (70 mujeres) no han tenido sintomatología, pero han recibido tratamiento el 5,7\%.

Tabla 7. Relación entre el consumo de tranquilizantes recetados y no recetados con el consumo de drogas

\begin{tabular}{|c|c|c|c|c|c|c|}
\hline $\begin{array}{l}\text { No } \\
\text { Sí }\end{array}$ & & & & $\begin{array}{l}\text { Con } \\
\text { susta }\end{array}$ & $\begin{array}{l}\text { mo } \\
\text { cias }\end{array}$ & Total \\
\hline & No & Recuento & & 123 & 82 & 205 \\
\hline $\begin{array}{l}\text { Consumo tranquilizantes } \\
\text { recetados }\end{array}$ & $\%$ dentro de Consum & sustancias & $83,7 \%$ & $51,3 \%$ & $66,8 \%$ & \\
\hline Sí & Recuento & & 24 & 78 & 102 & \\
\hline $\begin{array}{l}\text { Recuento } \\
\text { Consumo sustancias }\end{array}$ & $\%$ dentro de Consum & sustancias & $16,3 \%$ & $48,8 \%$ & $33,2 \%$ & \\
\hline & & & 147 & 160 & 307 & \\
\hline Total & & $100,0 \%$ & $100,0 \%$ & $100,0 \%$ & & \\
\hline & No & Recuento & & & 146 & 291 \\
\hline & Consumo sustancias & $100,0 \%$ & & $91,8 \%$ & $95,7 \%$ & \\
\hline Consumo tranquilizantes & Sí & Recuento & & & 13 & 13 \\
\hline & Consumo sustancias & $0,0 \%$ & & $8,2 \%$ & $4,3 \%$ & \\
\hline & Recuento & & & & 159 & 304 \\
\hline Total & $\begin{array}{l}\text { Consumo sustancias } \\
100,0 \%\end{array}$ & & $100,0 \%$ & $100,0 \%$ & & \\
\hline
\end{tabular}

Fuente: Elaboración propia a partir del proyecto REINAC Ref. EDU2016-79322-R. 
Primero, sobre la relación entre el consumo de tranquilizantes recetados y el consumo de drogas, la tabla muestra los porcentajes sobre el total de 147 No y 160 Sí. El contraste chi-cuadrado sobre la independencia de las variables es significativo $(<0,0001)$, verificándose las hipótesis para poder aplicarlo (Tabla $N^{0} 5$ ). Así se determina una asociación positiva significativa entre haber consumido alguna sustancia y haber consumido alguna vez tranquilizantes recetados, y tiene un cociente de ventajas de $\theta=4,875$, que indica que la probabilidad de consumir tranquilizantes recetados casi 5 veces superior en las consumidoras que en las no consumidoras.

Considerando un total de 307 mujeres con respuestas válidas, las consumidoras representan el 52,1\% ( $=160)$, de las cuales, un 48,8\% (78) ha consumido o consume tranquilizantes recetados. Este porcentaje es de 16,3\% (24) en el grupo de no consumidoras (47,9\%, 147 mujeres).

Segundo, en la relación entre la relación del consumo de tranquilizantes NO recetados con el consumo de drogas, sobre el total de la columna (145 No y 159 Sí), se observa: el contraste chi-cuadrado sobre la independencia de las variables es significativo $(p=0,001)$, verificándose las hipótesis para poder aplicarlo (Tabla No5). Podemos determinar que existe una asociación positiva significativa entre ambas variables.

Considerando un total de 304 mujeres con respuestas válidas, las consumidoras representan el 52,3\% (159), de éstas el 8,2\% (13) ha consumido o consume tranquilizantes no recetados. Se obtiene un cociente de ventajas de $\theta=26,816$, que indica que la probabilidad de consumir tranquilizantes no recetados casi 27 veces superior en las consumidoras que en las no consumidoras.

\section{Discusión}

La dificultad que implica vivir privado/a de libertad se refleja, entre otros, en el altísimo porcentaje de mujeres $(73,2 \%)$ de la muestra total estudiada que refieren haber tenido o tienen alguna sintomatología autopercibida de salud mental (psicológica/psiquiátrica), siendo las más recurrentes los trastornos del estado de ánimo (depresión 44,8\% y ansiedad 42,9\%) y cognoscitivos $(42,3 \%)$. Teniendo en cuenta que este estudio se realiza en medio abierto (semilibertad), donde las condiciones del cumplimiento de condena son más favorables, es coherente que estos datos sobre la tipología de sintomatologías sean ligeramente inferiores (entre 5-7 puntos) a los analizados por Turbi y Llopis (2017) con mujeres dentro de prisión, no obstante, coinciden con los planteamientos de Islam-Zwart, Vick y Rawlins (2007) y Gavin (2014), aunque éstos no refieren trabajos específicos con mujeres. Una situación que resulta más significativa y preocupante teniendo en cuenta que el $64,3 \%$ de esas mujeres estudiadas indican no haber recibido ningún tipo de tratamiento.

La rotundidad de estos datos evidencia que son situaciones que se producen con mayor presencialidad en la población penitenciaria que en la población general, los que se corroboran con planteamientos de la Red de Organizaciones Sociales del Entorno Penitenciario -ROSEP(2015), siendo estos entre 2 y 7 veces por encima de la población general. La explicación sobre esta realidad puede deberse, en parte, a los efectos del confinamiento y la falta de control sobre la propia vida, que obstaculiza la toma de decisiones y la posibilidad de intervenir en la evolución personal, familiar o social de las circunstancias externas (Echeverri, 2010). También encontramos otras circunstancias como la de compartir la celda con otra u otras personas, algo que puede dar lugar a un aumento de los niveles de ansiedad y problemas de convivencia (Rangel, Gil Parra 
y Vicente Cuenca, 2007, p.13).Además, desde el enfoque de género, se entiende que hombres y mujeres viven, asumen roles y sienten las situaciones de distinto modo, en el que las mujeres perciben las mismas con mayor intensidad, dolor y frustración, especialmente la relación maternal y familiar (Añaños, Fernández y Llopis, 2013; Añaños, Burgos, Rodríguez, Turbi, y Llopis, 2017).

Por otra parte, los tratamientos para paliar la sintomatología $(35,7 \%)$, han sido preferentemente de tipo farmacológico $(34,4 \%)$ y psicológico (12,3\%). La intervención socioeducativa en la salud mental es ínfima, registrando un escaso $3.5 \%$ de las mujeres analizadas con sintomatología que han realizado un programa de atención en salud mental (PAEIM) dentro de prisión, representando un escaso $2,6 \%$ en la muestra total. Una tendencia mayoritaria hacia la medicalización o atención psicológica/psiquiátrica, que viene a confirmar el afrontamiento de la problemática casi exclusivamente de forma clínica. Se trata de perspectivas parciales que dificultan otras esferas complementarias como son el desarrollo de la autonomía, autoestima, de las capacidades, destrezas, habilidades, etc., para poder afrontar un proceso reinsertador adecuado (Gil Cantero, 2010; Del Pozo, y Añaños, 2012; Martín, Vila y De Oña, 2012; Galán, 2015), además de responder a las necesidades sanitarias (Wolf, 2013).

Respeto al consumo de sustancias el 51,6\% ha tenido o tiene problemas con las drogas y el $23,2 \%$ son adictas. El 40,32\% de las consumidoras de drogas presentan sintomatología y el $19,4 \%$ de las adictas tiene un diagnóstico de salud mental, lo cual podemos afirmar que presentan una patología dual (adicción más uno o más trastornos de salud mental), dato que en la muestra general representa un escaso $4,5 \%$ y que a su vez, resulta un porcentaje bajo frente al informe de internos con TMG -sin distinguir sexo- que formaban parte del PAIEM (SGIP, 2019) donde indicaban que el 38,7\% presentaba patología dual.

La incidencia de sintomatología y consumo de drogas afecta a más de la mitad de mujeres estudiadas, pero a falta de diagnóstico no se puede afirmar la asociación, si bien es una cuestión de interés que indica la importancia del tema, tal cual ya lo señalaban en un estudio similar Añaños, et al (2017). La falta de diagnóstico y su adecuado tratamiento puede desembocar en conflictos (López-Barrachina, Lafuente y García- Latas, 2007) o ambientes que influyen negativamente la seguridad y el funcionamiento de los equipos de intervención (Arroyo-Cobo y Ortega, 2009).

Otras investigaciones señalan la asociación entre ambas cuestiones, como el 26,3\% que destacaban Lukasiewicz et al. (2009), éstos sin diagnóstico; sin embargo, Carava-Sánchez y García-Jarillob (2020) relacionan los trastornos del estado de ánimo con el consumo de alcohol y otras drogas durante el cumplimiento de la pena en prisión.

En la investigación queda confirmada la asociación significativa entre los tratamientos de salud mental y la sintomatología psicológica/psiquiátrica percibida, así como el consumo de drogas y consumos de tranquilizantes recetados como no rectados, siendo la probabilidad de consumir tranquilizantes recetados casi 5 veces superior en el grupo de consumidoras respecto a las no consumidoras y, siendo casi 27 veces superior en el caso de los tranquilizantes no recetados. García Rodicio (2019), refiere que los tranquilizantes sin receta son la segunda droga de abuso más importante después del cannabis, una información también reforzada por Mapelli-Caffaera, Herrera-Moreno y Sordi-Stock (2013), donde establecen que los tranquilizantes son el medicamento con mayor incidencia de consumo entre las mujeres privadas de libertad, actuando como medio de evasión y ocupación del tiempo (Bengoa, Karim y Errasti, 2012). 


\section{Conclusiones}

La prisión, además de ser un entorno punitivo, debe ser entendido no sólo como un espacio importante para abordar las necesidades y/o problemas, entre otras, de salud de estos grupos vulnerables, sino fundamentalmente un espacio educativo y reinsertivo. Los datos analizados indican situaciones que afectan a la mayor parte de las mujeres estudiadas con una determinada sintomatología asociada a un problema salud mental, que son escasamente diagnosticadas al igual que participan tímidamente en programas específicos; una situación parecida ocurre con el consumo de drogas y la asociación de ambas.

Las situaciones que rodean los problemas de la salud mental y los efectos del consumo de sustancias representan limitaciones o dificultades en la persona y en sus procesos de reinserción social, para lo cual es importante la identificación temprana y los consecuentes diagnósticos. Todo esto muestra la urgencia y necesidad de implementar programas socioeducativos y psicoeducativos que aborden de forma integral estas cuestiones, que vayan más allá de una atención medicalizada o de corte exclusivamente psicológica/psiquiátrica. Para ello, también es necesaria la presencia de especialistas en este ámbito. Actualmente no existe la figura del educador social en nuestras prisiones y su inmersión es fundamental para conseguir la recuperación de las personas privadas de libertad a partir de alternativas de intervención socioeducativa con perspectivas orientadas a la reducción de los factores de riesgo y a favorecer el desarrollo de los potenciales y factores de protección, teniendo en cuenta la perspectiva de género y en consonancia con la realidad que tienen que afrontar cuando adquieran la libertad.

Por último y a modo de prospectiva, resulta necesario aumentar este tipo de investigaciones para poder mejorar la situación de este colectivo en los entornos penitenciarios. Para ello, consideramos fundamental desarrollar investigaciones de carácter longitudinal, donde además, se tenga en cuenta a la población masculina con el propósito de obtener una visión lo más realista posible de la población penitenciaria que permita diseñar programas específicos que aborden la situación desde múltiples perspectivas, teniendo como referencia el género y la acción socioeducativa.

\section{Agradecimientos}

Agradecemos la financiación de este trabajo al Ministerio de Economía, Industria y Competitividad, la Agencia Española de Investigación y FEDER. Agradecemos el aval de la Secretaría General de Instituciones Penitenciarias y la Consejería de Justicia de la Generalitat de Catalunya. Proyecto $\mathrm{I}+\mathrm{D}+\mathrm{i}$ "Procesos de reinserción y acompañamiento a mujeres en semilibertad" (REINAC), Ref. EDU2016-79322-R, I.P. Fanny T. Añaños.

\section{Referencias}

Añaños, F., Fernández, P. y Llopis, J. (2013). Aproximación a los contextos en prisión. Una perspectiva socioeducativa. Pedagogía Social. Revista Interuniversitaria, 22, 13-28. DOI:http://dx.doi.org/10.7179//PSRI_2013.22.02

Añaños, F. (2017). Definición de los perfiles de adicción, y rasgos de consumo en mujeres reclusas. En Añaños, F. (coord.), En prisión. Realidades e intervención socioeducativa y drogodependencias en mujeres, (pp. 37-55). Madrid: Editorial Narcea. 
Añaños-Bedriñana, F., Burgos Jímenez, R., Rodríguez Sanjuán, A., Turbi Pizano y LlopisLlacer, J.J. (2017). Salud mental en prisión. Las paradojas socioeducativas. EduPsykhé, 16(1), 98-116 American Psychiatric Association (2014). Manual diagnóstico y estadístico de los trastornos mentales. Madrid: Médica Panamericana.

Arroyo Cobo, J.M. y Ortega, E. (2009). Los trastornos de personalidad en reclusos como factor de distorsión de clima social de la prisión. Revista Española de Sanidad Penitenciaria, 11, 11-15. Baillargeon, J., Penn, J. V., Knight, K., Harzke, A. J., Baillargeon, G. y Becker, E. A. (2010). Risk of reincarceration among prisoners with co-occurring severe mental illness and substance use disorders. Administration and Policy in Mental Health and Mental Health Services Research, 37(4), 367-374. DOI: 10.1007/s10488-009-0252-9

Bengoa, A., López, V., Karim Haidar, M. y Errasti, A. (2012). Drogodependencia entre muros. En I, Markez y C, Iñigo (Coord.), Guía. Atención y tratamientos en prisión por el uso de drogas (pp.15-25). Bilbao: OMEditorial

Boysen, G. A. (2007). Anevaluation of the DSM concept of mental disorder. The Journal of Mind and Behavior, 28(2), 157-173.

Butler, T., Indig, D., Allnutt, S. y Mamoon, H. (2011). Co-occurring mental illness and substance use disorder among Australian prisoners. Drug and Alcohol Review, 30(2), 188-194.DOI: 10.1111/j.1465-3362.2010.00216.x.

Caravaca-Sánchez, F. y García-Jarillob, M. (2020). Alcohol, otras Drogas y Salud Mental en Población Femenina Penitenciaria. Anuario de Psicología Jurídica, 30(1), 47-53. DOI: https://doi.org/10.5093/apj2019a15

Casas, M. y Guardia, J. (2002). Patología psiquiátrica asociada al alcoholismo. Adicciones, 14, 195-219. DOI: https://doi.org/10.20882/adicciones.524

Coronado Hurtado, T. (2016). Diagnóstico médico. Biociencias, 11(1), 69-73

Del Pozo Serrano, F. y Añaños-Bedriñana, F. (2012). La Educación Social penitenciaria: ¿De dónde venimos y hacia dónde vamos? Revista Complutense de Educación, Vol. 24, No 1 , 47-68. DOI:http://dx.doi.org/10.5209/rev_RCED.2013.v24.n1.41191

Echeverri Vera, J.A. (2010). La prisionalización, sus efectos psicológicos y su evaluación. Revista Pensando Psicología, 6 (11), 157-166.

Espinosa, M.I., y Laliga, A. (2005). Evolución del gasto farmacéutico en centros penitenciarios de Cataluña. Comunicaciones V Congreso Nacional de la Sociedad Española de Sanidad Penitenciaria. Revista Española de Sanidad Penitenciaria, 7, 49.

Ezama Coto, E., Alonso, Y. y Fontanil Gómez, Y. (2010). Pacientes, síntomas, trastornos, organicidad y psicopatología. International Journal of Psychology and PsychologicalTherapy,10(2), 293-314.

Galán, D. (2015). Los Módulos de Respeto: Una alternativa al tratamiento penitenciario (tesis doctoral). Madrid: Universidad Complutense de Madrid.

García Rodicio, S. (2019). Antídotos en centros penitenciarios. Boletín de Antídotos en Catalunya, $2(2), 1-6$.

Gavin, H. (2014). Criminological and Forensic Psychology. London: SAGE.

Gil Cantero, F. (2010). La acción pedagógica en las prisiones. Posibilidades y límites. Revista Española de Pedagogía, 68(245), 49-64.

Gil Extremera, B. (2019). Signos, síndromes y enfermedades con nombre propio. Educación Médica, 20(1), 165-168. DOI: https://doi.org/10.1016/j.edumed.2017.03.020 
Herrera, R. (2000). Salud mental y prisiones. Revista Española de Sanidad Penitenciaria, 2(2), 138-140.

Islam-Zwart, K.A. y Vik, P.W. (2004). Female adjustment to incarceration as influenced by sexual assault history. Criminal Justice and Behavior, 31, 521-541. DOI: https://doi. org/10.1177\%2F0093854804267091

López-Barrachina, R., Lafuente, O. y García-Latas, J. L. (2007). Del mito de Narciso a los trastornos de la personalidad en las cárceles aragonesas: Una aproximación al perfil de estos desórdenes en las personas privadas de libertad. Revista Española de Sanidad Penitenciaria, $9(2), 28-40$.

Lukasiewicz, M., Blecha, L., Falissard, B., Neveu, X., Benyamina, A., Reynaud, M. y Gasquet, I. (2009). Dual diagnosis: prevalence, risk factors, and relationship with suicide risk in a nation wide sample of French prisoners. Alcoholism: Clinical and Experimental Research, 33(1), 160-168. DOI: 10.1111/j.1530-0277.2008.00819.x

Mapelli-Caffarena, B., Herrera-Moreno, M. y Sordi-Stock, B (2013). La exclusión de las excluidas. ¿Atiende el sistema penitenciario a las necesidades de género? Una visión andaluza. Estudios Penales y Criminológicos, 33, 59-95.

Martín, V. M., Vila, E. S. y de Oña, J. M. (2012). La investigación educativa en el ámbito de las instituciones penitenciarias: panorámica, desafíos y propuestas. Revista de Educación, 360, 16-35. DOI: 10.4438/1988-592X-RE-2013-360-218

Ministerio de Sanidad, Servicio Sociales e Igualdad. (2016). Encuesta sobre salud y consumo de drogas en internados en instituciones penitenciarias. Recuperado de: http://www.pnsd.msssi. gob.es/profesionales/sistemasInformacion/sistemaInform acion/encuestas_ESDIP.htm

OMS. (2020). Trastornos mentales. Recuperado de: https://www.who.int/topics/mental_ disorders/es/

Rangel, F.B., Gil Parra, M. y Vicente Cuenca, M.A. (2007). Efectos Aparejados por el hecho de compartir celda. Percepción que tienen los internos sobre el hecho de compartir celda y los efectos aparejados en la población reclusa de los Centros Penitenciarios de la Comunidad de Madrid. Revista de Estudios Penitenciarios, 253, 9-29.

Ruiz Narezo, M. y Santibáñez Gruber, R.M. (2014). Prisión, drogas y educación social. Educació Social. Revista d'Intervenció Socioeducativa, 57, 118-134.

ROSEP (2015). Estudio de la realidad penal y penitenciaria: una visión desde las entidades sociales. Recuperado de:https://plataformarosep.files.wordpress.com/2016/04/estudio-dela-realidad-penal-y-penitenciaria-una-visic3b3n-desde-las-entidades-sociales.pdf

Secretaría General de Instituciones Penitenciarias (2020). Programa de Intervención con Enfermos Mentales. Recuperado de http:/www.institucionpenitenciaria.es/web/portal/Reeducacion/ ProgramasEspecificos/enfermosMentales.html

Secretaría General de Instituciones Penitenciarias (2019). Informe General 2018. Ministerio del Interior. Secretaría General Técnica. Recuperado de http://www.institucionpenitenciaria.es/ web/export/sites/default/datos/descargables/publicaciones/Informe_General_2018_acc.pdf

Turbi, A. y Llopis, J. (2017). Salud física y mental en mujeres reclusas en las cárceles españolas. En Añaños, F., En prisión. Realidades e intervención socioeducativa y drogodependencias en mujeres (pp. 71-86). Madrid: Narcea. 
Vega-Dienstmaier, J. (2017). ¿Debe mejorar la forma de diagnosticar en psiquiatría? Revista de Neuropsiquiatria 80 (3), 155-157. DOI: http://dx.doi.org/10.20453/rnp.v80i3.3151

Villagrá P., González A., Fernández P., Casares M.J., Rodríguez F., Martín J.L. (2011). Perfil adictivo, delictivo y psicopatológico de una muestra de mujeres en prisión. Adicciones, 23, 219-26.DOI: https://doi.org/10.20882/adicciones.146

Wolf, H. (2013). Opiate Substitution Treatment and Harm Reduction in prisons: the Geneva model. Recuperado de: https://rm.coe.int/mental-health-and-addiction-in-prisons-writtencontributions-to-the-in/168075f532 